\title{
Protection against Divergent Influenza H1N1 Virus by a Centralized Influenza Hemagglutinin
}

\author{
Eric A. Weaver ${ }^{1 *}$, Adam M. Rubrum ${ }^{2}$, Richard J. Webby ${ }^{2}$, Michael A. Barry ${ }^{1,3,4,5}$ \\ 1 Division of Infectious Diseases, Department of Internal Medicine, Mayo Clinic, Rochester, Minnesota, United States of America, 2 Department of Infectious Diseases, \\ St Jude Children's Research Hospital, Memphis, Tennessee, United States of America, 3 Department of Immunology, Mayo Clinic, Rochester, Minnesota, United States of \\ America, 4 Department of Molecular Medicine, Mayo Clinic, Rochester, Minnesota, United States of America, 5 Translational Immunovirology and Biodefense Program, \\ Mayo Clinic, Rochester, Minnesota, United States of America
}

\begin{abstract}
Influenza poses a persistent worldwide threat to the human population. As evidenced by the $2009 \mathrm{H} 1 \mathrm{~N} 1$ pandemic, current vaccine technologies are unable to respond rapidly to this constantly diverging pathogen. We tested the utility of adenovirus ( $\mathrm{Ad}$ ) vaccines expressing centralized consensus influenza antigens. Ad vaccines were produced within 2 months and protected against influenza in mice within 3 days of vaccination. Ad vaccines were able to protect at doses as low as $10^{7}$ virus particles/kg indicating that approximately 1,000 human doses could be rapidly generated from standard Ad preparations. To generate broadly cross-reactive immune responses, centralized consensus antigens were constructed against $\mathrm{H} 1$ influenza and against $\mathrm{H} 1$ through $\mathrm{H} 5$ influenza. Twenty full-length $\mathrm{H} 1 \mathrm{HA}$ sequences representing the main branches of the $\mathrm{H} 1 \mathrm{HA}$ phylogenetic tree were used to create a synthetic centralized gene, HA1-con. HA1-con minimizes the degree of sequence dissimilarity between the vaccine and existing circulating viruses. The centralized H1 gene, HA1-con, induced stronger immune responses and better protection against mismatched virus challenges as compared to two wildtype $\mathrm{H} 1$ genes. HA1-con protected against three genetically diverse lethal influenza challenges. When mice were challenged with 1934 influenza A/PR/8/34, HA1-con protected $100 \%$ of mice while vaccine generated from 2009 A/TX/05/09 only protected $40 \%$. Vaccination with $1934 \mathrm{~A} / \mathrm{PR} / 8 / 34$ and $2009 \mathrm{~A} / \mathrm{TX} / 05 / 09$ protected $60 \%$ and $20 \%$ against 1947 influenza A/FM/1/47, respectively, whereas $80 \%$ of mice vaccinated with HA1-con were protected. Notably, $80 \%$ of mice challenged with 2009 swine flu isolate A/California/4/09 were protected by HA1-con vaccination. These data show that HA1-con in Ad has potential as a rapid and universal vaccine for H1N1 influenza viruses.
\end{abstract}

Citation: Weaver EA, Rubrum AM, Webby RJ, Barry MA (2011) Protection against Divergent Influenza H1N1 Virus by a Centralized Influenza Hemagglutinin. PLoS ONE 6(3): e18314. doi:10.1371/journal.pone.0018314

Editor: Anil Tyagi, University of Delhi, India

Received December 29, 2010; Accepted February 24, 2011; Published March 28, 2011

Copyright: (C) 2011 Weaver et al. This is an open-access article distributed under the terms of the Creative Commons Attribution License, which permits unrestricted use, distribution, and reproduction in any medium, provided the original author and source are credited.

Funding: Work conducted at St Jude Children's Research Hospital was funded by the US National Institute of Allergy and Infectious Diseases, National Institutes of Health, under Contract No. HHSN266200700005C. The funders had no role in study design, data collection and analysis, decision to publish, or preparation of the manuscript.

Competing Interests: The authors have declared that no competing interests exist.

*E-mail: weaver.eric@mayo.edu

\section{Introduction}

Annually, $5-15 \%$ of the world's population is affected by influenza epidemics and have upper respiratory tract infections, 3 to 5 million have severe illness and 250,000 to 500,000 cases result in death [1]. While these normal infections are certainly of concern, natural pandemic influenza outbreaks and intentional releases of pathogenic influenza are of substantially higher concern. A difficult obstacle inherent to current trivalent inactivated vaccine (TIV) production stems from the need to screen and predict which viruses may circulate in the subsequent year. These predictions do not always accurately identify the actual viruses that cause disease that year $[2,3,4]$. In fact, vaccine mismatches occurred in 4 out of $8(50 \%)$ flu seasons in the USA between 1997 and 2005 [5,6,7,8]. In addition to this problem, some of the immunologic effects of TIV reduces its efficacy. For example, the current vaccine only provides short-term immunity [9], the induced immunity is highly strain specific [3,10], intramuscular delivery does not stimulate high levels of the secretory IgA that is less specific and is more reactive against heterologous viruses $[10,11,12,13,14]$, and fail to induce crossprotective cellular immunity $[15,16,17,18,19]$.

In addition to influenza virus based vaccines, alternative approaches have been investigated. These include the use of electroporated DNA expression plasmids, adenovirus vectored vaccines, and universal vaccines based on centralized genes and conserved matrix ectodomains [20,21,22,23,24]. Centralized genes were first proposed as universal vaccines for HIV $[25,26,27,28,29,30]$. Centralized sequences minimize the degree of dissimilarity between a vaccine strain and contemporary circulating viruses by creating an artificial sequence based on the most common amino acid in each position in an alignment. Other than HIV, centralized genes have also been proposed as universal vaccines for highly pathogenic $(\mathrm{H} 5 \mathrm{Nl})$ avian influenza $(\mathrm{HPAI})$ and Chikungunya virus [20,31,32].

Recently, swine flu was declared a pandemic. Even with the latest technologies the $\mathrm{CDC}$ and the $\mathrm{WHO}$ were not able to agree on a vaccine strategy and implement vaccine production in time for the 2009-2010 influenza season [33]. Due to the delay in vaccine availability, the CDC has estimated that between April 


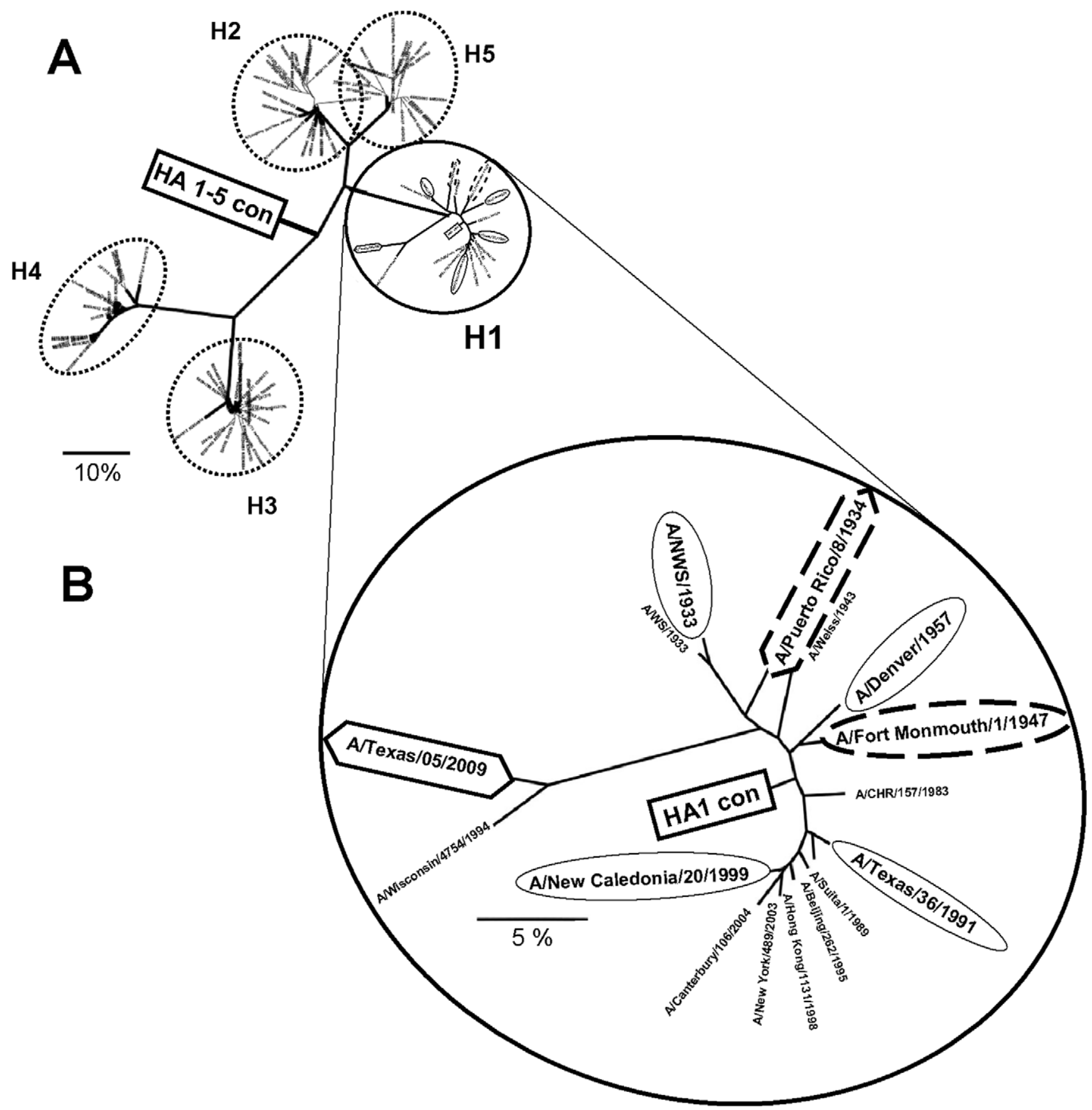

Figure 1. Phylogenetic trees showing the wildtype genetic relationship of wildtype and consensus HA genes. Selected full-length $\mathrm{H} 1$ $\mathrm{HA}$ sequences were downloaded from NCBI and aligned using Clustal W. The 566 amino acid consensus sequences was determined from this alignment. The HA 1-5 con gene is shown localized to the central region of the multi-subtype phylogenetic tree (A). The HA1-con gene is shown in the phylogenetic $\mathrm{H} 1$ subtype tree (B). Unrooted phylogenetic trees were created using PHYLIP version 3.5c. The boxes represent consensus genes used to create the adenovirus vaccines. Diamonds represent wildtype gene used to create adenovirus vaccines. The circles represent viruses or peptides used to evaluate in vitro immune correlates induced by the vaccines. Dashed lines represent viruses used in lethal challenges. doi:10.1371/journal.pone.0018314.g001

and November $19^{\text {th }} 2009$ there were between 34 and 67 million cases of $2009 \mathrm{H} 1 \mathrm{~N} 1,154,000$ and 303,000 2009 H1N1-related hospitalizations, and 7,070 and 13,930 $2009 \mathrm{H} 1 \mathrm{N1}$-related deaths [34]. $2009 \mathrm{H} 1 \mathrm{Nl}$ has also been confirmed in more than 276 pediatric deaths to date. More than double the annual seasonal flu pediatric deaths [34].

To address the issue of pandemic $\mathrm{H} 1 \mathrm{Nl}$ influenza virus, we created a centralized $\mathrm{Hl} \mathrm{HA}$ immunogen. When in vitro immune correlates of protection and in vivo prophylaxis was compared. We found that the centralized $\mathrm{H} 1$ antigen, $\mathrm{HAl}$-con, induced greater and broader immune correlates as compared to two wildtype $\mathrm{Hl}$ antigens. We also found that in the case of vaccine mismatch HAlcon could induce more potent protection against lethal wildtype influenza challenges. Based on these data, this strategy may be applicable to the more divergent $\mathrm{H} 1 \mathrm{Nl}$ influenza viruses and represents an alternative universal vaccine that could be either 
stockpiled or included in the current vaccine formulations in the case of $\mathrm{H} 1$ seasonal or pandemic mismatch.

\section{Materials and Methods}

\section{Alignment, Consensus, and Tree Construction}

Influenza sequence databases contain a tremendous amount of sequence information. There are over 30,000 HA sequences available through Genbank. Although valuable, not all of this information can be used to create a consensus sequence. During pandemics, epidemics and endemics there is a renewed interest in influenza evolution. At these times multiple nearly identical influenza sequences are entered into the databases from specific institutions diluting out more divergent, but less sequenced isolates. Therefore a consensus sequence created using all of the available sequence information would bias the consensus sequence to that of the most sequenced isolates and those closely genetically related. To reduce this bias, we selected twenty full-length $\mathrm{H} 1 \mathrm{HA}$ sequences from the larger sequence space to represent the main branches of the H1. (accessions: ISDN13422, CY001952, CY003833, GY002392, CY006363, CY007467, CY002624, CY003384, CY003304, CY003696, AF386780, AF386775, AY289928, AF386774, D13574, X17221, U02085, M38312, S62154, and U53162). Novel 2009 H1N1 HA genes were not included in the initial H1 HA consensus design because they had not been identified at the time of analysis. Selected full-length $\mathrm{H} 1$ HA sequences were downloaded from Genbank and aligned using Clustal W. All aligned sequences were then inspected manually to correct for apparent mistakes. Positions containing gaps or ambiguously aligned positions were removed from the datasets. The 566 amino acid consensus sequence, named HAl-con, was generated from this alignment by using the most common amino acid at each position. Unrooted phylogenetic trees were created using PHYLIP version 3.5c (Figure 1B). The HA 1-5 consensus gene, HA 1-5 con, was created by the same strategy with the exception that sequences from $\mathrm{H} 1, \mathrm{H} 2, \mathrm{H} 3$ and $\mathrm{H} 5$ were used (Figure 1A) (accessions: D13574, S62154, U02085, NC_002017, CY002624, GY006363, AF386775, AF386780, AY289928, ISDN13422, GY003833, CY002392, GY003384, GY007467, CY003696, AY209963, AY209961, L20409, L11134, D13579, L1 1 126, L1 1 142, L20406, L1 1125, M54895, AJ289703, V01103, CY006044, CY003064, M55059, CY002056, DQ249261, CY000137, CY002136, AY032978, AB019357, CY002088, X05907, GY002072, CY002904, AJ252131, CY000017, CY003512, GY002744, CY002496, ISDN121986, AB239125, DQ372591， ISDN119678, ISDN117778, ISDN117777, ISDN1 18371, AJ867074, ISDN1 10940, AY555150, ISDN40341, AY651334, AY651335, ISDN40278, AY575869, AY575870, ISDN38262, AF102676, AF084279, AF046097, AF084280, and AF084532). $\mathrm{N}$ and $\mathrm{O}$ linked glycosylation sites for the vaccine genes HAl-con, A/PR/8/34, and A/TX/05/09 were analyzed using NetNGlyc 1.0 and NetOGly 3.1 software analyses.

\section{Viruses and Vaccines}

Influenza virus $\mathrm{A} / \mathrm{PR} / 8 / 34$ was obtained from ATCG (VR95). Influenza viruses A/Denver/1/57, A/Fort Monmouth/1/47, A/ Texas/36/91, and A/NWS/33 were obtained from the Biodefense and Emerging Infections Research Resources Repository. All of the viruses were passaged one time in SPF embryonated eggs and the chorioallantoic fluid was stored at $-80 \mathrm{C}$. The pandemic $\mathrm{H} 1 \mathrm{~N} 1$ virus used in challenge studies was a mouse-adapted A/ California/04/09 virus. This virus was adapted for lethality in mice through serial lung passage as previously described [35].
Stocks of virus were grown in embryonated eggs and the chorioallantoic fluid was stored at $-80 \mathrm{C}$ ).

The influenza virus $\mathrm{A} / \mathrm{PR} / 8 / 34$ and $\mathrm{A} / \mathrm{FM} / 1 / 47$ stock was titered in $\mathrm{BALB} / \mathrm{c}$ mice to determine its $50 \%$ mouse lethal dose $\left(\mathrm{MLD}_{50}\right)$. The HA sequences for A/PR/8/34, A/TX/05/09 A/ $\mathrm{HK} / 213 / 03$ were obtained from NCBI and the Influenza Sequence Database at Los Alamos National Laboratories, respectively. HAl-con, HA 1-5 con, A/PR/8/34, A/TX/05/ 09, and A/HK/213/03 HA genes were codon-optimized for mammalian expression and synthesized by Genscript. Inc. First generation replication defective (E1/E3 deleted) Ad5 vectors were constructed using the Ad-Easy system in 293A cells as described in [36]. All adenoviruses were purified by $\mathrm{CsCl}$ banding and quantitated by OD260. The recombinant Adenoviruses expressing $\mathrm{A} / \mathrm{PR} / 8 / 34, \mathrm{~A} / \mathrm{TX} / 05 / 09, \mathrm{~A} / \mathrm{HK} / 213 / 03$, HAl-con and HAl5 con were named Ad-PR-HA, Ad-TX-HA Ad-HK-HA, AdHA1-con, and Ad-HA-1-5-con, respectively. The infectivity of the recombinant adenoviruses was checked using the Adeno-X Rapid titer. There were no statistical differences in infectivity (Figure S1). All virus stocks were found to contain $\leq 0.1 \%$ replication competent adenovirus.

\section{Animals}

Female BALB/c mice (6-8 weeks old) were purchased from Charles River Laboratories (Wilmington, Massachusetts, USA) and housed in the Mayo Clinic or St Jude Children's Research Hospital Animal Facility under the Association for Assessment and Accreditation of Laboratory Animal Care (AALAC) guidelines with animal use protocols approved by the corresponding the Mayo Clinic Institutional Animal Care and Use Committee (IACUC protocol No. A1 10). All animal experiments were carried out according to the provisions of the Animal Welfare Act, PHS Animal Welfare Policy, the principles of the NIH Guide for the Care and Use of Laboratory Animals, and the policies and procedures of Mayo Clinic and St Jude Children's Research Hospital.

Mice were anesthetized i.p. with ketamine $(140 \mathrm{mg} / \mathrm{kg}) /$ kylazine $(5.55 \mathrm{mg} / \mathrm{kg})$ and were immunized intramuscularly (i.m.) with various doses of Adenovirus in a volume of $50 \mathrm{ul}$. Twenty-five $\mu$ l was injected into each mouse quadriceps. Three weeks post-immunization the mice were challenged intranasally with mouse-adapted influenza virus $\mathrm{A} / \mathrm{PR} / 8 / 34, \mathrm{~A} / \mathrm{FM} / 1 / 47$, or A/California/04/09.

\section{Cellular Immunity: Enzyme-Linked Spot (ELISPOT) Assay}

Mice were immunized i.m. with $10^{10}$ virus particles (vp) of adenovirus expressing HA genes from PR, TX or H1 consensus. Three weeks post-immunization the mice were sacrificed and spleens were harvested. ELSIPOT assays were performed as previously described [37]. Overall cellular immune responses were measured using three pools of a peptide array, Influenza Virus A/ New Caledonia/20/99 (H1Nl) Hemagglutinin Protein. The peptides consisted of 9416 - to 17-mers, with 11 or 12 amino acid overlaps and were obtained from the Biodefense and Emerging Infections Research Resources Repository (Catalog No. NR-2602). Then using a matrix of peptide pools and individual peptides the immuno-stimulatory epitopes were mapped. Concanavalin A $(5 \mu \mathrm{g} / \mathrm{ml})$ was used as a positive control while splenocytes from DPBS immunized animals as well as media only wells were used as negative controls. Responses were considered as positive if the number of the spots was four-fold higher than that of the negative control and at least $50 \mathrm{SFC} / 10^{6}$ cells. 
Table 1. Clustal Distance Matrix.

\begin{tabular}{llllllll}
\hline & & & & & & & \\
\hline & $\mathbf{A} /$ FM/1/47 & A/NWS/33 & A/TX/36/91 & A/Denver/1/57 & $\begin{array}{l}\text { A/New Caledonia } \\
\text { /20/99 }\end{array}$ & A/PR/8/34 & A/CA/04/09 \\
\hline A/PR/8/34 & 0.097 & 0.078 & 0.122 & 0.115 & 0.126 & 0.000 & 0.186 \\
A/TX/05/09 & 0.196 & 0.182 & 0.208 & 0.202 & 0.202 & 0.186 & 0.005 \\
A/HK/213/03 & 0.374 & 0.362 & 0.369 & 0.317 & 0.368 & 0.355 & 0.371 \\
HA1-con & $\mathbf{0 . 0 4 8}$ & $\mathbf{0 . 0 8 3}$ & $\mathbf{0 . 0 5 5}$ & $\mathbf{0 . 0 7 8}$ & $\mathbf{0 . 0 5 9}$ & $\mathbf{0 . 0 8 5}$ & $\mathbf{0 . 1 7 3}$ \\
HA 1-5 con & $\mathbf{0 . 3 1 7}$ & $\mathbf{0 . 3 1 3}$ & $\mathbf{0 . 3 1 3}$ & $\mathbf{0 . 3 6 6}$ & $\mathbf{0 . 3 0 6}$ & $\mathbf{0 . 3 1 5}$ & $\mathbf{0 . 3 1 8}$ \\
\hline
\end{tabular}

Distance matrix calculated using ClustalW with no exclusions of positions with gaps and no correction for multiple substitutions.

doi:10.1371/journal.pone.0018314.t001

\section{Humoral Immunity: Hemaglutination Inhibition (HI) Assay}

Mice were immunized i.m. with $10^{10}$ vp of adenovirus expressing HA genes from $\mathrm{PR}, \mathrm{TX}$ or $\mathrm{Hl}$ consensus. Three weeks post-immunization the mice were bled by cardiac puncture and sacrificed. Serum was collected using Becton Dickinson microtainer tubes with serum separator. Starting at a dilution of 1:5 sera were diluted two-fold in $50 \mu \mathrm{l}$ of DPBS in a 96-well, nonsterile, nontissue culture-treated, round bottom microtiter plate. Four HAU of influenza virus in $50 \mathrm{ul}$ was added to the diluted sera and incubated at room temperature (RT) for $1 \mathrm{hr}$. After incubation, $50 \mu \mathrm{l}$ of a $1 \%$ chicken RBC solution was added and incubated at RT for $1 \mathrm{hr}$. The HI titer was determined to be the highest serum dilution to inhibit hemagglutination.

\section{Influenza Challenge}

Mice were immunized with various doses of the Ad-vectored vaccines. Three weeks after immunization the mice were anesthetized i.p. with ketamine $(140 \mathrm{mg} / \mathrm{kg}) /$ xylazine $(5.55 \mathrm{mg} /$ $\mathrm{kg})$. The mice were weighed for baseline measurements. The mice were challenged intranasally with $100 \mathrm{LD}_{50}$ of influenza $\mathrm{A} / \mathrm{PR} / 8$ / 34 or $\mathrm{A} / \mathrm{FM} / 1 / 47$ virus or $70 \mathrm{LD}_{50}$ of $\mathrm{A} /$ California/04/09 virus. The mice were placed on their backs and $10 \mu \mathrm{l}$ of $\mathrm{A} / \mathrm{PR} / 8 / 34$ or $\mathrm{A} / \mathrm{FM} / 1 / 47$ virus was pipetted into each nare for a total volume of $20 \mu \mathrm{l}$. For infection with A/California/04/09, $15 \mu \mathrm{l}$ was delivered to each nare for a total of $30 \mu \mathrm{l}$. The mice were then weighed and monitored daily for signs of disease. Mice were humanely euthanized if their body weight dropped to $75 \%$ of baseline weights.

\section{Results}

\section{Production of Centralized Consensus HA Immunogens}

A comparison of select $\mathrm{Hl}$ hemagglutinin (HA) protein sequences from 1933 through 2009 generated a phylogenetic tree with $\sim 21.0 \%$ of sequence divergence across the branches (Figure 1B). Due to high levels of genetic diversity, selecting a single wildtype HA protein as a universal vaccine is not thought to be feasible. Rather than select one wildtype gene as a vaccine, we generated a centralized gene that mimics an ancestor of influenza infections during the past 76 years. The rationale for this approach is to produce an immunogen that is centrally located with respect to all other variants. Such a protein then practically has lower sequence divergence with all of the variants than any two randomly selected genes.

Phylogenetic analysis shows that the synthetic centralized HA1con protein localizes to the central region of the $\mathrm{Hl}$ tree (Figure 1B). When genetic distances were calculated using ClustalW, the HAl-con was found to be half the genetic distance to the wildtype influenza strains used in this study (Table 1). For example, influenza A/FM/1/47 was used as a challenge strain in subsequent studies using three homologous vaccines, HAl-con, A/ $\mathrm{TX} / 05 / 09$ and $\mathrm{A} / \mathrm{PR} / 8 / 34$. When the genetic distances from the mismatched vaccines to the challenge strain were calculated, the HAl-con was closest genetically with $4.8 \%$ divergence. A/TX/ 05/09 and A/PR/8/34 were found to be $19.6 \%$ and $9.7 \%$ divergent, respectively (Table 1$)$. When vaccinated mice were challenged with $\mathrm{A} / \mathrm{PR} / 8 / 34$ the mismatched vaccines $\mathrm{HAl}$-con and $\mathrm{A} / \mathrm{TX} / 05 / 09$ were $8.5 \%$ and $18.6 \%$ divergent, respectively. Again, HAl-con is closest genetically when the vaccine and challenge strain are mismatched. Although HAl-con was not half the genetic distance to the A/CA/04/09 flu challenge strain as compared to the mismatched $\mathrm{A} / \mathrm{PR} / 8 / 34$, it was slightly closer (Table 1).

Alignment of HA amino acid sequences showed that the HAlcon protein conserved functional elements that include cleavage, fusion, transmembrane and cytoplasmic domains (Figure 2). Comparison of predicted $\mathrm{N}$ and $\mathrm{O}$ linked glycosylation sites for HAl-con to HAs from A/PR/8/34 and A/TX/05/09 indicated that all of the N-glycosylation sites for A/TX/05/09 and A/PR/ 8/34 were present in HAl-con (Figure 2). In addition, there were two additional potential $\mathrm{N}$-glycosylation sites in the HAl-con at positions 144 and 201. The predicted $\mathrm{N}$-glycosylation at position 144 was also found in several other isolates such as $\mathrm{A} / \mathrm{FM} / 1 / 47$ and $\mathrm{A} / \mathrm{CHR} / 157 / 83$. The predicted $\mathrm{N}$-glycosylation at position 201 in HAl-con was not present in the subset of HA proteins analyzed, but represents a conserved asparagine sequence from the $20 \mathrm{HA}$ proteins used for construction. No O-glycosylation was predicted in HAl-con, A/PR/8/34, or A/TX/05/09 (Figure 2).

A broader HA consensus protein (HA 1-5 con) was engineered as a centralized immunogen spanning $\mathrm{H} 1, \mathrm{H} 2, \mathrm{H} 3, \mathrm{H} 4$, and $\mathrm{H} 5$ influenza viruses (Figure 1A). Although H4 influenza infections have yet to be discovered in humans, it was included in the tree construction to illustrate that the HA 1-5 con gene localizes centrally to this subtype as well. Influenza has a tremendous amount of evolutionary plasticity in its HA sequences as $\mathrm{H} 3$ and $\mathrm{H} 5$ proteins, for example, are as much as 60\% divergent (Figure 1A). A blast search of the Genbank viral sequences database of the HA 1-5 con protein sequence revealed the closest influenza isolates had only $69 \%$ identity. All of the closest isolates were $\mathrm{H} 1 \mathrm{Nl}$ viruses and included A/SouthCarolina/1/1918 (H1N1), A/swine/Jamesburg/1942 (H1N1), A/swine/Iowa/ 1945 (H1N1), and A/duck/Italy/281904/2006 (H1N1). Table 1 shows that the genetic distance between HA 1-5 con and all of selected $\mathrm{H} 1$ isolates is equally distant from them as the $\mathrm{H} 5$ influenza virus A/Hong Kong/213/2003 (A/HK/213/03). Based on conventional wisdom, the amino acid divergence of this synthetic HA 1-5 con gene would not be predicted to induce 


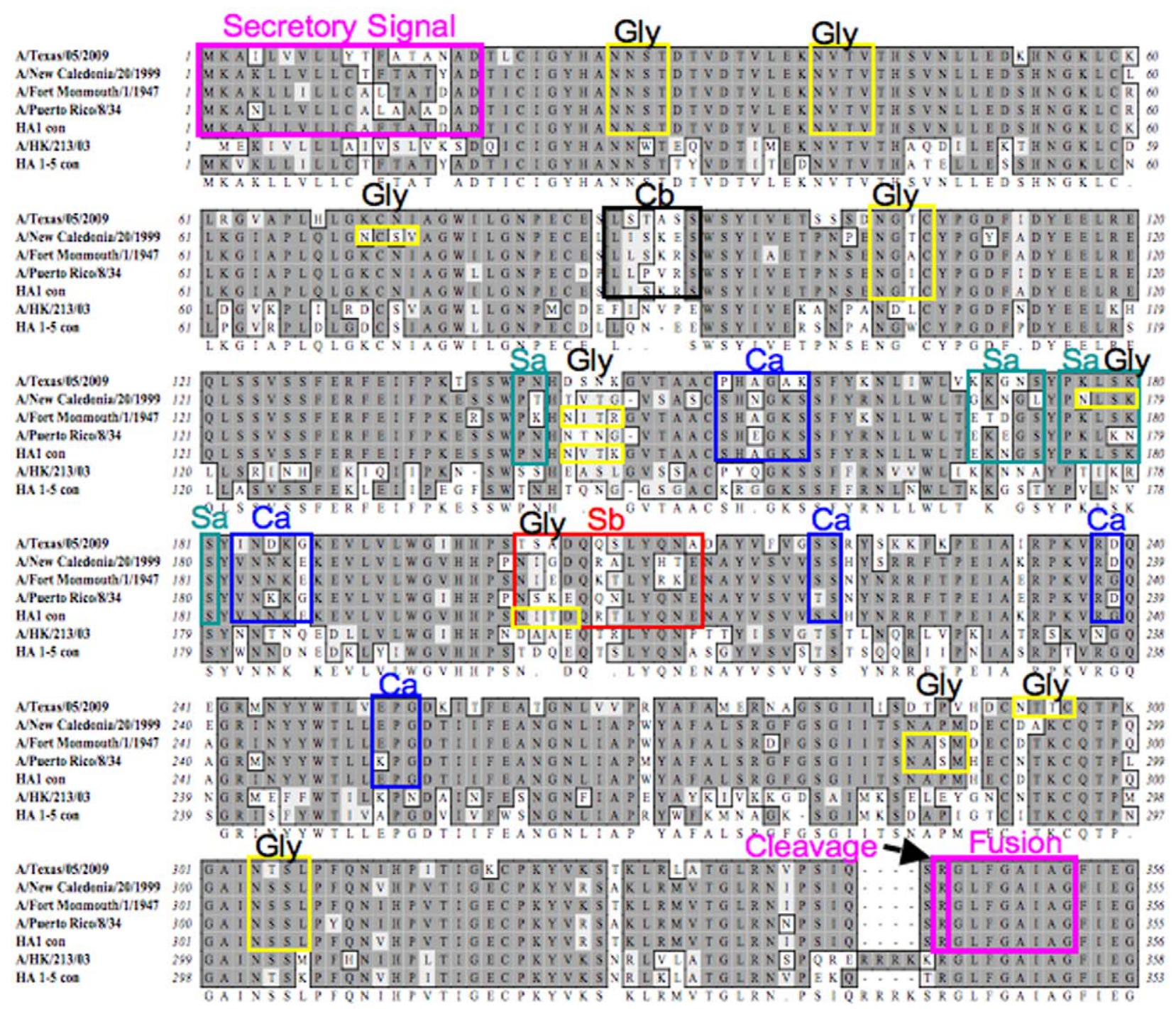

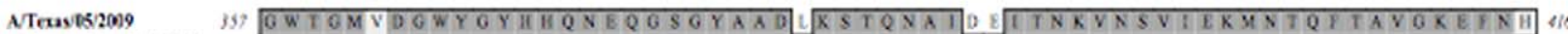

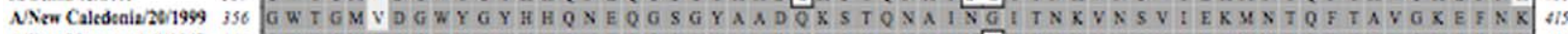

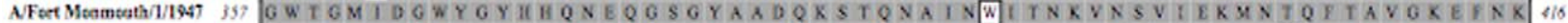

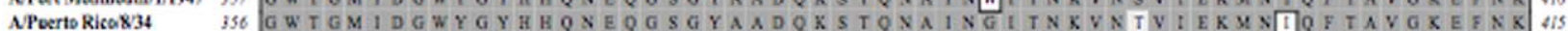

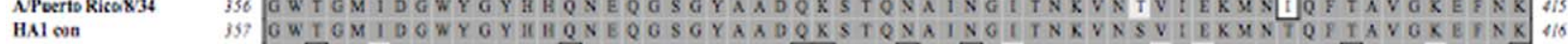

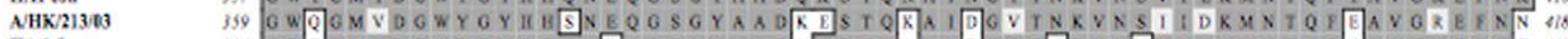

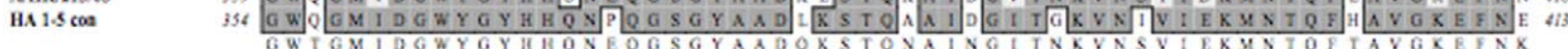

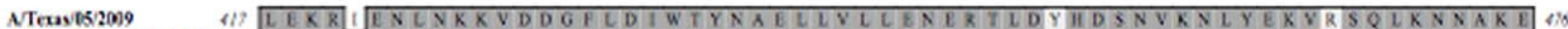

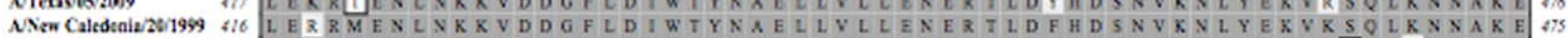

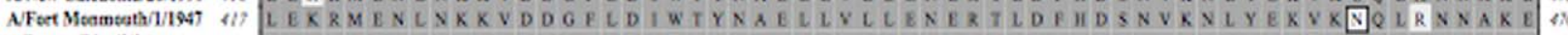

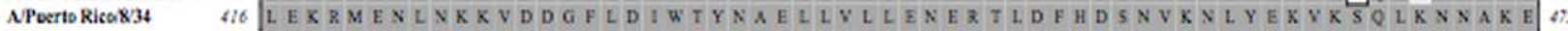

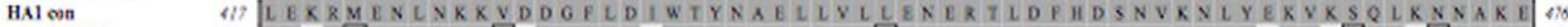

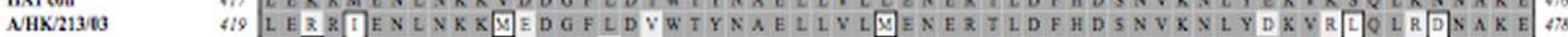

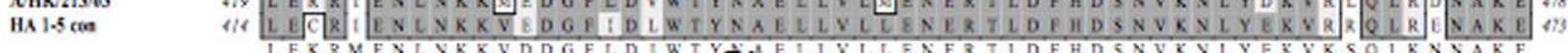

\section{Gly}

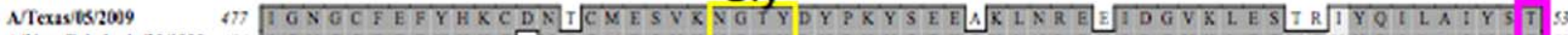

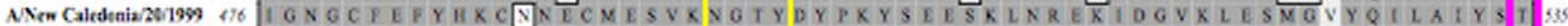

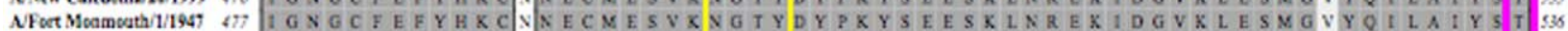

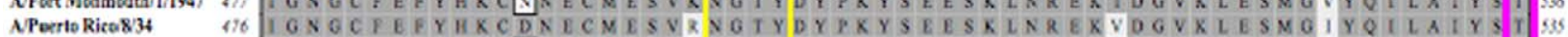

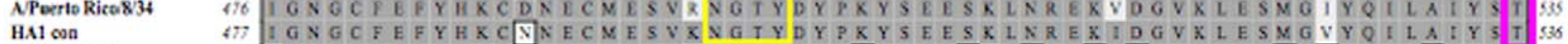

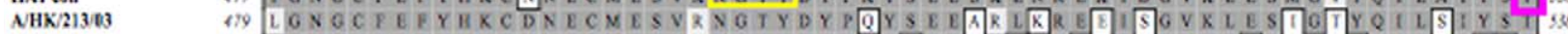

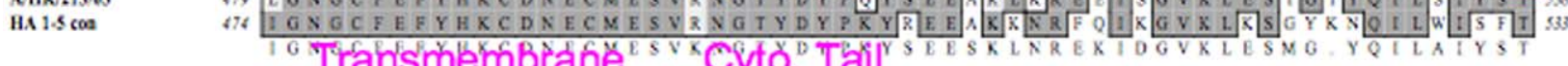

Nexas $16 / 2099$

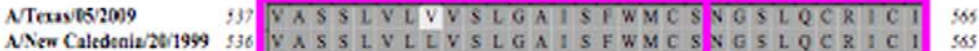

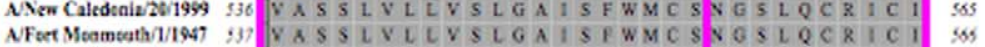

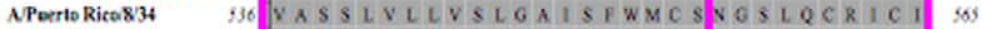

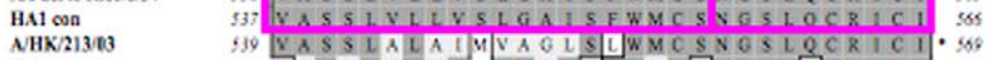

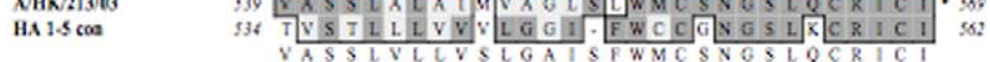


Figure 2. Alignment of the centralized influenza vaccine genes, the wildtype virus genes and the A/New Caledonia/20/99 HA proteins showing the location and sequence of the major antigenic sites $\mathbf{S a}, \mathbf{S b}, \mathbf{C a}$ and $\mathbf{C b}$. The conserved functional elements consisting of the secretory signal, cleavage, fusion, transmembrane and cytoplasmic tail are indicated and boxed. N-linked glycosylation sites (Gly) are shown boxed in yellow.

doi:10.1371/journal.pone.0018314.g002

protection against $\mathrm{Hl}$ influenzas. Based on this, a codonoptimized gene for HA 1-5-con was constructed and used to generate the vaccine Ad HA 1-5-con. This vector was included as a divergent immunogen to act as a likely negative control for the HAl-con vaccine, but also as a long-shot cross-reactive vaccine against many influenza viruses.

\section{Intrasubtype Protection at High Doses of Vaccine}

Mice were immunized with $10^{10}$ virus particles (vp) of all of the Ad influenza vaccines. Three weeks later, the immunized mice received a lethal challenge with one hundred times the mean lethal dose (100 $\mathrm{LD}_{50}$ ) of influenza $\mathrm{A} / \mathrm{PR} / 8 / 34$ virus (Figure $3 \mathrm{~A}$ ). Only HAl-con and the cognate immunogen $\mathrm{A} / \mathrm{PR} / 8 / 34 \mathrm{HA}$ were able to protect mice from disease, weight loss, and death. A/TX/05/09 HA was able to protect the mice from death. However, there were still signs of disease and weight loss using a large dose of vaccine. As predicted the other vaccines were unable to protect against disease and death.

\section{Protection Against Homologous Challenge at Low Dose}

Direct comparison of gene-based vaccines in non-human primates has demonstrated that Ad vaccines generate more robust immune responses and protection than plasmid or vaccinia vaccines [38]. Given our goal to develop a gene-based vaccine for influenza, we tested this vaccine platform by inserting the codon-optimized cDNA for HA from $\mathrm{Hl}$ influenza A/Puerto Rico/8/1934 (A/PR/8/34) into a E1-deleted replication-defective Ad5 vector (Ad-PR-HA). To titrate the efficacy of the vaccine, groups of 5 female $\mathrm{BALB} / \mathrm{c}$ mice were immunized with a range of doses of Ad-PR-HA by the intramuscular (i.m.) route and were challenged 3 weeks later with $100 \mathrm{LD}_{50}$ of influenza A/PR/8/34 (Figure 3B). A vaccine dose of $10^{7}$ vp was capable of completely protecting mice from death and disease. An even lower dose of $10^{6}$ vp demonstrated reduced weight loss and increased survival against this stringent lethal challenge.

\section{Rapid Protection Against Stringent Lethal Challenge}

Ad vectors produce rapid and high levels of protein production in vivo [39]. To assess the kinetics of Ad vaccine protection, groups of $5 \mathrm{BALB} / \mathrm{c}$ mice were vaccinated with $10^{10} \mathrm{vp}$ of Ad-PR-HA by the i.m. route and then challenged with $100 \mathrm{LD}_{50}$ of $\mathrm{A} / \mathrm{PR} / 8 / 34$ influenza 1, 3, or 5 days after vaccination (Figure 3C). Under these conditions, mice were fully protected from this challenge within 5 days of vaccination. In addition, mice vaccinated 3 days before challenge showed clinical signs of disease and weight loss, but most survived this stringent challenge. These data suggest that Ad vaccines may generate rapid protection against influenza.

\section{Cross-reactive Cellular Immune Responses}

Three weeks after i.m. immunization with $10^{10} \mathrm{vp}$ of recombinant adenovirus, the mice were sacrificed and splenocytes were harvested for ELISPOT assays (Figure 4A and B). Splenocytes were stimulated with overlapping peptides from the heterologous virus A/New Caledonia/20/99 (Figure S2). Both A/PR/8/34 and HA1con immunized mice induced significantly greater cellular immune responses than A/TX/05/09 immunized mice with $\mathrm{p}$ values of 0.026 and 0.001 , respectively (Figure 4A). Individual immunostimulatory peptides were identified using overlapping peptide pools and individual peptides (Figure 4B). Greater than 50 interferon- $\gamma$ spot-forming cells (SFC) were considered significant. Epitope mapping revealed that five epitopes $(6,21 / 22,31,78$ and 90) were recognized by splenocytes from the immunized mice (Figure 4B). HAl-con generated greater $\mathrm{T}$ cell responses against all peptides than either A/PR/8/34 or A/TX/05/09 with the only exception being against peptide 6 . T cells from HAl-con immunized mice reacted against two dominant epitopes 78 and 90 while $\mathrm{A} / \mathrm{PR} / 8 / 34$ only induced one dominant $\mathrm{T}$ cell response against epitope 90 . In addition HAl-con immunized mice induced $\mathrm{T}$ cell responses against epitope 31 while A/PR/8/34 and A/TX/05/09 immunized mice did not. Interestingly, almost all of these epitopes were entirely conserved in all of the genes (Figure S2). There were two amino acid substitutions in epitope 31 that were not in HAl-con. HAl-con immunized mice induce much stronger $\mathrm{T}$ cell immune responses against epitope 78 , however there were no sequence differences between the HAl-con and A/PR/8/34 and A/TX/05/ 09 at that site.

\section{Cross-reactive Humoral Immune Responses}

Groups of $10 \mathrm{BALB} / \mathrm{c}$ were immunized with $10^{10} \mathrm{vp}$ of $\mathrm{Ad}$ vaccine. Three weeks after immunization, sera from the mice were tested for hemagglutination inhibition (HI) antibody responses against a series of wildtype $\mathrm{Hl}$ influenza viruses (Figure 4G). HAlcon induced $\mathrm{HI}$ titers that were equal or greater than those induced by $\mathrm{A} / \mathrm{PR} / 8 / 34$ or $\mathrm{A} / \mathrm{TX} / 05 / 09$ against isolates $\mathrm{A} / \mathrm{FM} /$ 1/47, A/TX/36/1991 and A/NWS/33. HAl-con immunized mice generated significantly higher $\mathrm{HI}$ titers against $\mathrm{A} / \mathrm{FM} / 1 / 47$ influenza than $\mathrm{A} / \mathrm{PR} / 8 / 34(\mathrm{p}=<0.001)$. HAl-con induced significantly greater $\mathrm{HI}$ titers against $\mathrm{A} / \mathrm{FM} / 1 / 47$ and $\mathrm{A} / \mathrm{NWS}$ / 33 viruses than A/TX/05/09 $(\mathrm{p}=<0.001)$. However, A/TX/05/ 09 did induce significantly higher $\mathrm{HI}$ titers against A/Denver/1/ 57 than both Ad-PR-HA and Ad-HAl-con $(p=<0.001)$. Values were $\log$ transformed for statistical analyses.

\section{Protection Against Lethal A/PR/8/34 Influenza}

As expected the homologous $\mathrm{A} / \mathrm{PR} / 8 / 34$ vaccine was able to induce protective responses using the lowest dose of vaccine as compared to the mismatched vaccines, HAl-con and A/TX.05/ 09 (Figure 5). A/PR/8/34 immunized mice did not show any signs of disease or death with doses as low as $10^{7}$ vp (Figure 5). Immunization with $10^{6} \mathrm{vp}$ did not prevent disease, but did provide protection against disease in $75 \%$ of mice. Doses lower than $10^{6} \mathrm{vp}$ resulted in disease and death (Figure S3). In regard to the vaccine mismatches, the HAl-con was able to protect against disease at a dose of $10^{8} \mathrm{vp}$ while A/TX/05/09 vaccinated mice exhibited signs of disease and weight loss. Both HAl-con and A/TX/05/09 were able to protect against death at a dose of $10^{8} \mathrm{vp}$. Mice vaccinated with HAl-con at a dose of $10^{7} \mathrm{vp}$ did show signs of disease and weight loss. However, these mice recovered and survived the challenge whereas $60 \%$ of mice vaccinated with $\mathrm{A} /$ TX/05/09 at the same dose did not survive (Fig 5) $(p=0.05)$. Doses lower than $10^{7}$ vp resulted in disease and death (Figure S3).

\section{Protection Against Lethal A/FM/1/47 Influenza}

In contrast to the homologous $\mathrm{A} / \mathrm{PR} / 8 / 34$ virus challenge, $\mathrm{A} /$ $\mathrm{PR} / 8 / 34 \mathrm{HA}$ was not able to induce protection from disease and death from lethal A/FM/1/47 at a dose of $10^{7}$ vp (Figure 6). In fact, doses up to $10^{9} \mathrm{vp}$ of Ad-PR-HA were unable to prevent 

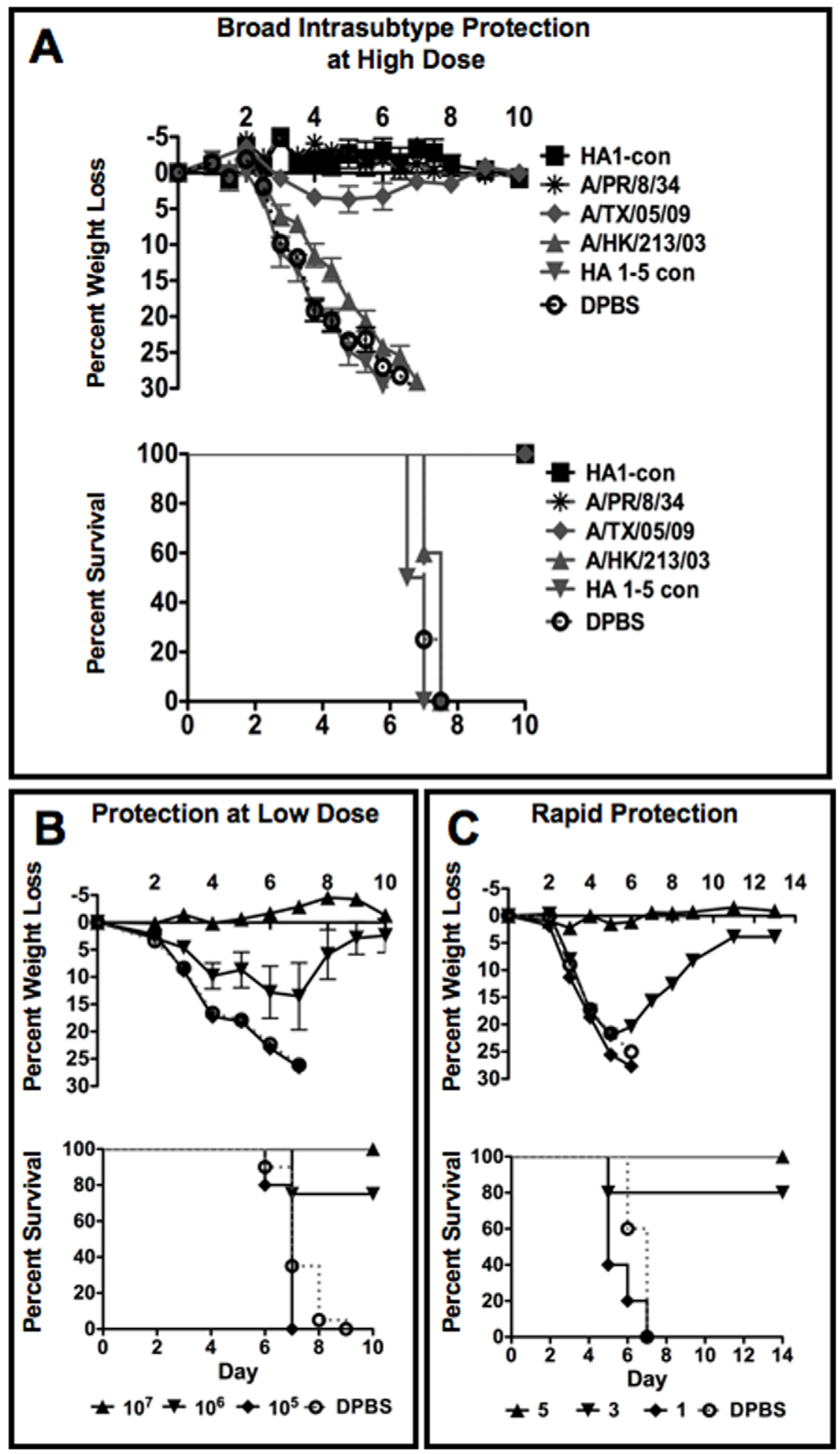

Figure 3. Adenovirus as a platform for Influenza vaccines. Prophylactic responses in mice immunized with consensus and wildtype adenoviral vectors were determined by immunizing mice with $10^{10} \mathrm{Ad}$ vaccine viral particles. Three weeks after immunization the mice were challenged intranasally with $100 \mathrm{MLD}_{50}$ of influenza $\mathrm{A} / \mathrm{PR} / 8 / 34$ virus (A). In order to determine how much ad vaccine would be required to induce prophylaxis, mice were immunized intramuscularly with 10 -fold dilutions of adenovirus expressing the A/PR/8/34 HA and challenged with $100 \mathrm{MLD}_{50}$ of influenza $\mathrm{A} / \mathrm{PR} / 8 / 34$ virus 3 weeks later (B). In order to determine the length of time to induce prophylaxis and the duration of prophylactic immune responses using Ad vaccines, mice were immunized intramuscularly with $10^{10} \mathrm{vp}$ of $\mathrm{Ad}$ expressing A/PR/8/34 HA. The mice were challenged intranasally with $100 \mathrm{MLD}_{50}$ of influenza A/PR/8/34 virus 1, 3, 5 and 200 days post immunization (C). Weight loss and survival were monitored daily. Mice that lost more than $25 \%$ of their baseline weight were humanely sacrificed.

doi:10.1371/journal.pone.0018314.g003 

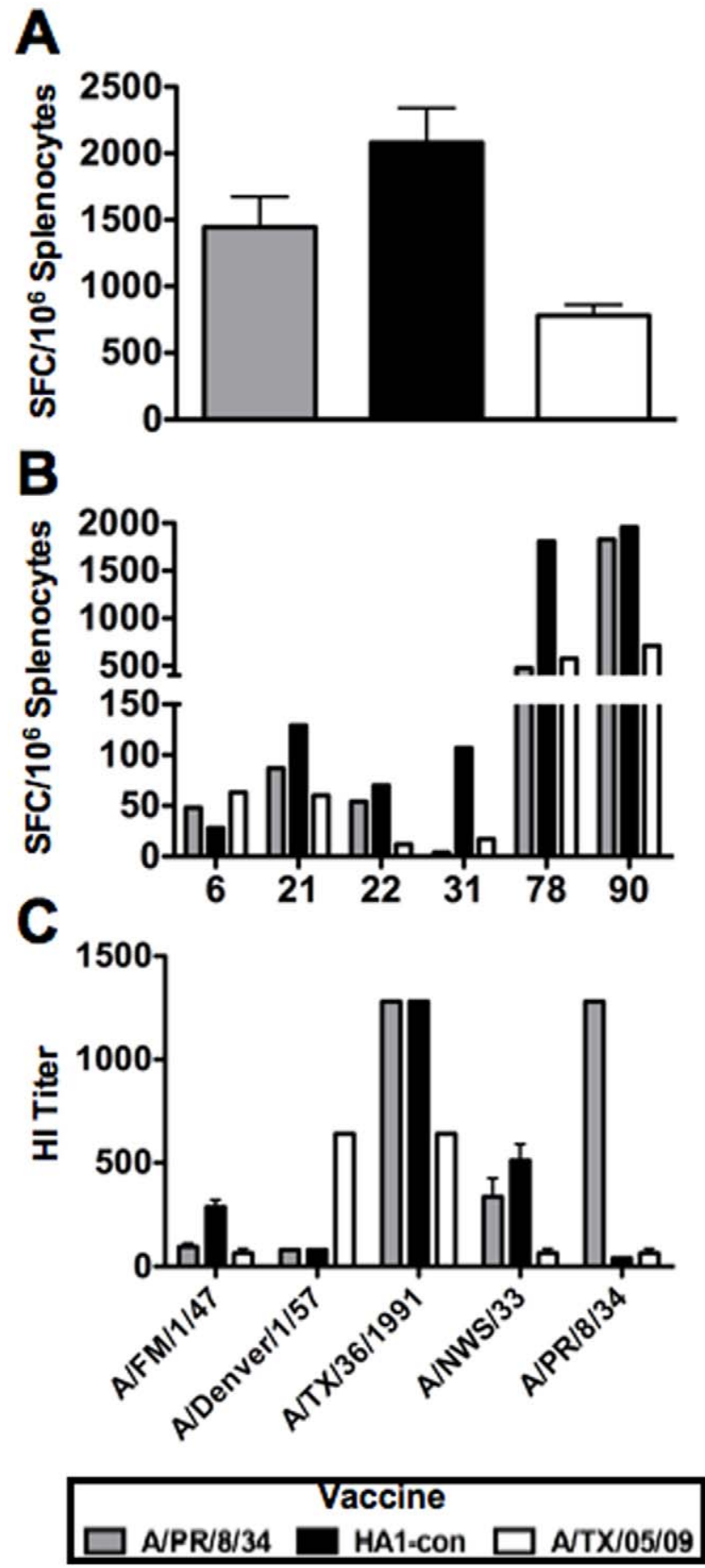

Figure 4. Immune correlates of Protection. Mice were immunized with $10^{10} \mathrm{Ad}$ vaccine viral particles. Three weeks post-immunization the mice were bled and spleens were removed for anti-influenza humoral and cellular responses. Splenocytes were stimulated with peptide pools representing full-length A/New Caledonia/20/99 HA protein in order to determine the overall magnitude of wildtype cellular immune responses (A). Individual peptides were used to identify specific epitopes recognized by splenocytes from immunized mice. The peptide numbers that resulted in positive cellular responses are shown (B). Hemagglutination inhibition $(\mathrm{HI})$ titers induced by the vaccine genes against wildtype viral isolates (C). Groups of 5 mice were used and error bars represent standard error.

doi:10.1371/journal.pone.0018314.g004 disease and weight loss (Figure S4). The mismatched A/TX/05/ 09 vaccine was even less effective at inducing protective responses against the influenza A/FM/1/47 virus lethal challenge. Doses of $10^{9} \mathrm{vp}$ of A/TX/05/09 were unable to protect against death. However, Ad-HAl-con was able to induce protection against weight loss at a dose of $10^{9} \mathrm{vp}$ (Figure S4). Vaccination with a dose of $10^{8} \mathrm{vp}$ of HAl-con provided the best protection against weight loss and death (Figure 6). Both HAl-con and A/PR/8/34 protected against death while A/TX/05/09 did not resulting in only $60 \%$ survival. A lower dose, $10^{7} \mathrm{vp}$, resulted in weight loss and death in all groups, however, the HAl-con group were best protected resulting in less average weight loss and the best survival (Figure 6). Mice immunized with $10^{7}$ vp of HAl-con resulted in $80 \%$ protection against death whereas only $20 \%$ of A/TX.05/09 immunized mice were protected from death $(p=0.06)$. Doses lower than $10^{7} \mathrm{vp}$ resulted in significant weight loss and death in all groups. However, the only mouse to survive after immunization with $10^{6}$ vp was in the HAl-con group (Figure S4).

\section{Protection Against Lethal A/California/04/09}

As predicted the A/TX/05/09 vaccine provided the best levels of protection against a lethal challenge with A/CA/04/09 (Figure 7). Similarly, A/TX/05/09 protected against death at a dose of $10^{7} \mathrm{vp}$ and provided some protection at a dose of $10^{6} \mathrm{vp}$ as $\operatorname{did} \mathrm{A} / \mathrm{PR} / 8 / 34$ against a homologous lethal challenge. A/TX/ 05/09 vaccine provided complete protection against weight loss and disease at a dose of $10^{8}$ vp (Figure S5). When the vaccines were mismatched, the centralized HAl-con proved to be better at inducing protective immunity as compared to A/PR/8/34 (Figure 7). At higher doses HAl-con vaccinated mice showed less average weight loss and were better protected against death than A/PR/8/34 vaccinated mice resulting in $80 \%$ and $20 \%$ survival, respectively. While there were no significant differences in survival between mice immunized with $10^{10} \mathrm{vp}$ of either HAl-con or A/ TX/05/09, there were significant differences between A/TX/05/ 09 and $\mathrm{A} / \mathrm{PR} / 8 / 34$ survival rates $(\mathrm{p}=0.01)$. At a dose of $10^{9} \mathrm{vp}$ $60 \%$ of HAl-con vaccinated mice survived while only $20 \%$ of A/ $\mathrm{PR} / 8 / 34$ vaccinated mice survived (Figure 7 ). There were no statistical differences in survival between mice immunized with $10^{9}$ vp of A/TX/05/09 or HAl-con. However, A/PR/8/34 ad A/ TX/05/09 immunized mice did have significantly different survival rates $(\mathrm{p}=0.01)$. All doses lower than $10^{9} \mathrm{vp}$ of mismatched vaccine resulted in death (Figure S5).

\section{Discussion}

We tested a centralized gene for use as a universal vaccine against $\mathrm{H} 1 \mathrm{~N} 1$ influenza viruses that might be relevant to seasonal and pandemic influenza. Phylogenetic analysis of HAl-con showed that it did localize to the central region of the phylogenetic tree and was, for the most part, genetically equidistant to the majority of wildtype isolates. In vitro characterization showed that an adenoviral vectored HAl-con gene could induce immune correlates in mice equal to or greater than that induced by wildtype genes. Results from the ELISPOT assay indicates that $\mathrm{HAl}$-con induces stronger and broader $\mathrm{T}$ cell immune responses in splenocytes. While these cellular immune responses are the primary means by which an influenza viral infection is cleared they are not essential to prevent infection. Humoral immune responses as measured by the $\mathrm{HI}$ assay indicate that HAl-con could induce protective responses against all four wildtype isolates tested, providing that the standard measure of protection is an $\mathrm{HI}$ titer of 40 or higher [40]. The immune correlates presented here are limited. Intranasal IgA and lung cellular immune responses are 


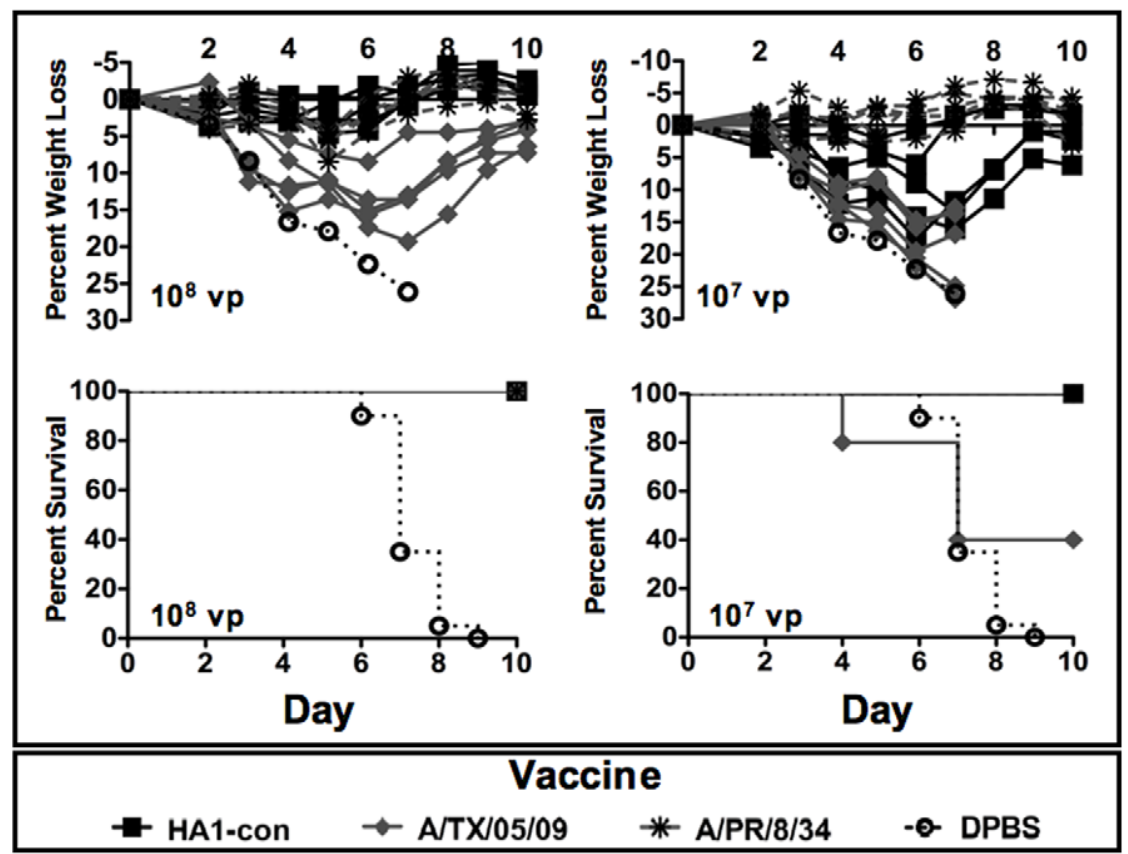

Figure 5. Protection against $A / P R / 8 / 34$ influenza virus infection. Mice were immunized intramuscularly with various doses of $A / P R / 8 / 34, A d-$ HA1-con, and A/TX/05/09 HA expressing virus. Three weeks after immunization the mice were challenged intranasally with $100 \mathrm{LD}_{50}$ of influenza virus A/PR/8/34. Individual mouse weights for the vaccinated mice and the mean and standard error of the control DPBS immunized mice are shown. Mice exhibiting profound signs of disease and less than $75 \%$ of baseline weights were humanely sacrificed. doi:10.1371/journal.pone.0018314.g005

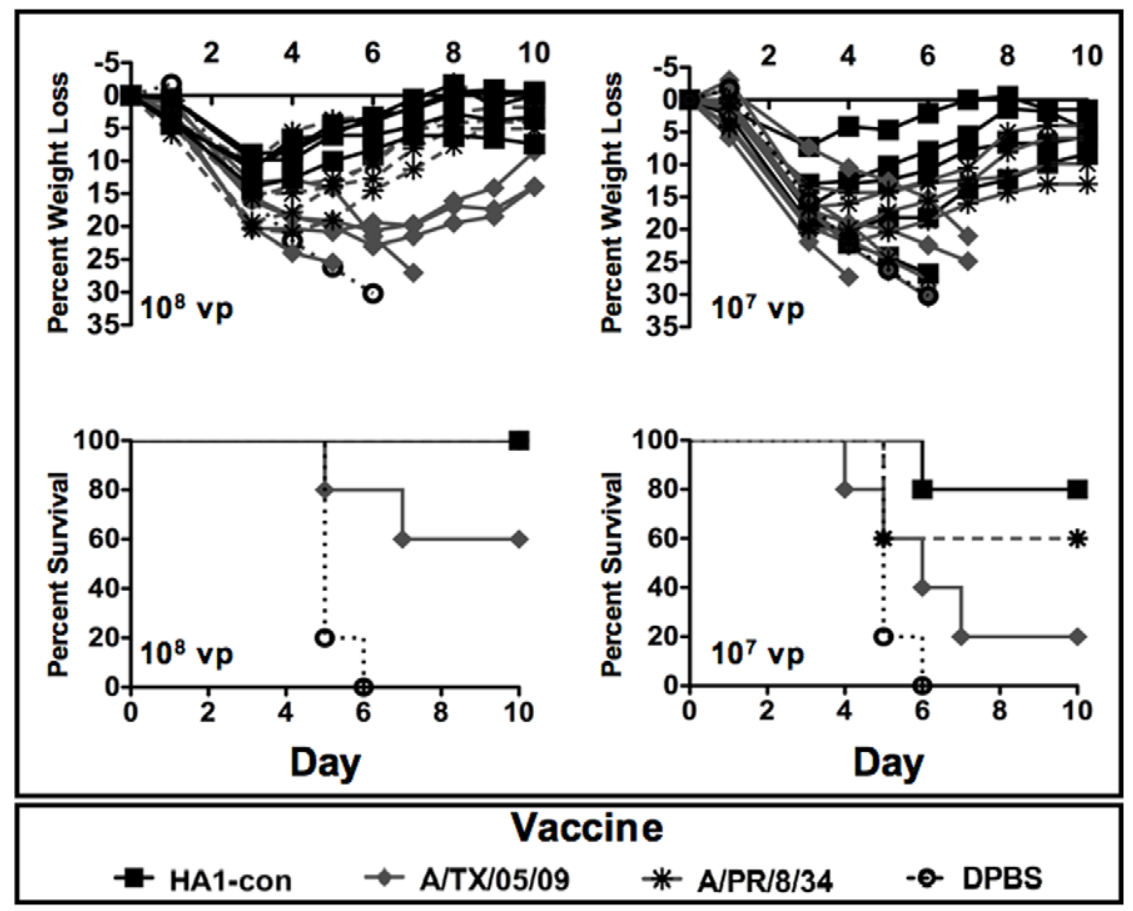

Figure 6. Protection against A/FM/1/47 influenza virus infection. Mice were immunized intramuscularly with various doses of $A / P R / 8 / 34, A d-$ $\mathrm{HA} 1$-con, and A/TX/05/09 HA expressing virus. Three weeks after immunization the mice were challenged intranasally with $100 \mathrm{LD}_{50}$ of influenza virus $\mathrm{A} / \mathrm{FM} / 1 / 47$. Individual mouse weights for the vaccinated mice and the mean and standard error of the control DPBS immunized mice are shown. Mice exhibiting profound signs of disease and less than $75 \%$ of baseline weights were humanely sacrificed.

doi:10.1371/journal.pone.0018314.g006 


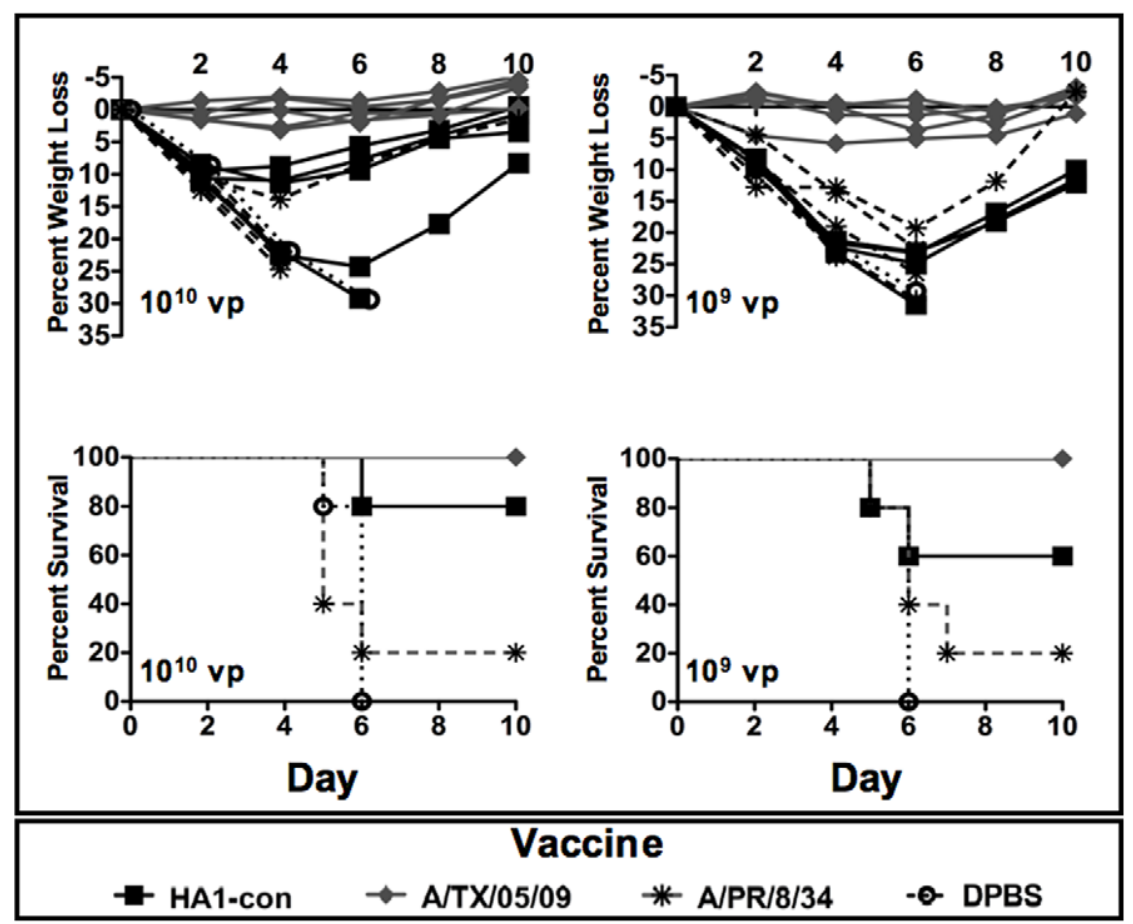

Figure 7. Protection against A/CA/04/09 influenza virus infection. Mice were immunized intramuscularly with various doses of $A / P R / 8 / 34$, Ad-HA1-con, and A/TX/05/09 HA expressing virus. Three weeks after immunization the mice were challenged intranasally with $70 \mathrm{LD}_{50}$ of influenza virus of $\mathrm{A} / \mathrm{CA} / 04 / 09$. Individual mouse weights for the vaccinated mice and the mean and standard error of the control DPBS immunized mice are shown. Mice exhibiting profound signs of disease and less than $75 \%$ of baseline weights were humanely sacrificed.

doi:10.1371/journal.pone.0018314.g007

also critical immune correlates of protection. However, since we planned to evaluate the vaccine-induced immune responses using a virus challenge advanced immune assays were not necessary. Also, the dose used for this initial evaluation was considerable and would not be practical if scaled up for use in humans.

The ultimate proof of a better vaccine lies in the data from the challenge studies. Not only did the HAl-con centralized gene induce protective responses against all three $\mathrm{H} 1 \mathrm{Nl}$ lethal challenges, it was a superior vaccine as compared to all other mismatch vaccines. Although HAl-con did provide better protection against A/California/04/09 than the mismatched A/ $\mathrm{PR} / 8 / 34$, these protective responses were at high doses of vaccine. However, these studies were done using high doses of pathogenic influenza virus. The use of $100 \mathrm{LD}_{50}$ is not representative of any epidemic or pandemic ever recorded. Therefore, protective responses against infectious, but not lethal influenza virus may be achieved with even lower doses of adenovirus vectored vaccines. It was surprising to find that matched Ad vectored vaccines provided protection against a very stringent lethal challenge at very low doses. Doses as low as $10^{6}$ vp of Ad $\left(4 \times 10^{7} \mathrm{vp} / \mathrm{kg}\right)$ reduced weight loss and increased survival against stringent lethal challenge. Extrapolation of this vaccine dose to a $70 \mathrm{~kg}$ human suggests that Ad vaccine production from a simple cell factory of $10^{9}$ cells would produce as many as 1,000 estimated human doses of influenza vaccine. Given that it requires up to 3 eggs to produce one human dose of current trivalent vaccine, this suggests that Ad may be a viable platform for human vaccines. This combined with the observed rapid protection within 5 days of immunization make Ad an attractive influenza vaccine platform to combat seasonal and epidemic spread of influenza.

Another interesting aspect of this study was the creation and testing of a multi-subtype centralized gene, HA 1-5 con. One of the attractive qualities of a centralized immunogen is that conserved or "shared" regions or epitopes would be incorporated into the gene. However, it could be that in the case of high levels of divergence (i.e. inter-subtype) these conserved epitopes would be lost. From a viral evolution standpoint it is interesting that there do not appear to be intermediate HA sequences found in nature that are similar to the centralized HA 1-5 con. However, within the subtypes there are naturally occurring sequences that are similar to HAl-con. In addition to the absence of viral intermediates similar to the HA 1-5 con gene, it is at least $\sim 30 \%$ divergent from all reported wildtype isolates. Given $\mathrm{H} 5$ and $\mathrm{H} 1$ genes are similarly divergent and that $\mathrm{H} 5$ genes do not protect against $\mathrm{H} 1$ wildtype virus, it is very possible that HA 1-5 con may simply act as a control HA gene for further studies.

Although the centralized influenza genes proposed in this study are for use as human vaccines, the same concepts and perhaps the same genes may be used to vaccinate reservoir animals against influenza infections. Vaccination of reservoir animals at the source of virus evolution could intervene at this primary step and could result in the elimination of potential future influenza outbreaks that result in pandemics. To this end, our Ad virus vector platform may also be applicable in this approach and may prove to be more potent and cost-effective than traditional vaccines.

While previous studies have reported on the efficacy of consensus H5 genes conferring protection against wildtype influenza virus infection, this is the first reported study that has applied this concept to the more diverse $\mathrm{Hl}$ subtype. Here we show that HAl-con is a superior vaccine in the case of mismatch only and produced equal or greater cross-protective immune correlates against wildtype viruses as compared to wildtype HA gene vaccines. However, in the case of a matched vaccine the homologous vaccine gene is superior to HAl-con. For example, 
Ad-PR induces superior levels of protection against A/PR/8/34 virus as compared to the centralized HAl-con.These data support the concept of using consensus genes as influenza vaccines either as single vaccines or as a "platform" gene to provide broad immunologic cross-reactivity for combination with other single isolate influenza genes.

\section{Supporting Information}

Figure S1 In order to determine the quality of the adenoviral vectors being used the infectivity of the preps were analyzed using the AdenoX rapid titer kit. 293 cells were infected with each of the viral preps incubated overnight and stained for hexon expression. There were no significant differences in the Adenoviral vaccine preps. (TIF)

Figure S2 Alignment of the consensus influenza vaccine genes, the wildtype virus genes and the A/New Caledonia/20/99 HA proteins. Numbers represent the epitopes identified in Figure 4. Boxes represent the individual peptides that were recognized by immunized mice splenocytes. The green box represents a unique epitope recognized only by Ad-HAl-con immunized splenocytes and the red box represents the conserved immunodominant CTL epitope. Groups of 5 mice were used and error bars represent standard error. (TIF)

Figure S3 Dose-dependent prophylactic responses against a lethal $A / P R / 8 / 34$ influenza virus challenge. Mice were immunized intramuscularly with various doses of A/ $\mathrm{PR} / 8 / 34$, Ad-HAl-con, and A/TX/05/09 HA expressing virus. Three weeks after immunization the mice were challenged intranasally with $100 \mathrm{LD}_{50}$ of influenza virus $\mathrm{A} / \mathrm{PR} / 8 / 34$. Weight loss and death in mice immunized $10^{6} \mathrm{vp}$ are shown in $\mathrm{A}$ and $\mathrm{B}$, respectively. Weight loss and death in mice immunized $10^{5} \mathrm{vp}$ are shown in $\mathrm{C}$ and $\mathrm{D}$, respectively. The mean and standard error of the control DPBS immunized mice are shown. Mice exhibiting profound signs of disease and less than $75 \%$ of baseline weights were humanely sacrificed.

(TIF)

Figure S4 Dose-dependent prophylactic responses against a lethal $\mathrm{A} / \mathrm{FM} / 1 / 47$ influenza virus challenge.

\section{References}

1. WHO (2009) Influenza Fact Sheet.

2. Gitelman AK, Kaverin NV, Kharitonenkov IG, Rudneva IA, Zhdanov VM (1984) Changes in the antigenic specificity of influenza hemagglutinin in the course of adaptation to mice. Virology 134: 230-232.

3. Kilbourne ED, Smith C, Brett I, Pokorny BA, Johansson B, et al. (2002) The total influenza vaccine failure of 1947 revisited: major intrasubtypic antigenic change can explain failure of vaccine in a post-World War II epidemic. Proc Natl Acad Sci U S A 99: 10748-10752.

4. Schild GC, Oxford JS, de Jong JC, Webster RG (1983) Evidence for host-cell selection of influenza virus antigenic variants. Nature 303: 706-709.

5. (1998) Update: influenza activity-United States and worldwide, 1997-98 season, and composition of the 1998-99 influenza vaccine. MMWR Morb Mortal Wkly Rep 47: 280-284.

6. (2001) Update: influenza activity-United States and worldwide, 2000-01 season, and composition of the 2001-02 influenza vaccine. MMWR Morb Mortal Wkly Rep 50: 466-479.

7. (2003) Update: Influenza activity-United States, 2002-03 season. MMWR Morb Mortal Wkly Rep 52: 26-28.

8. (2004) Update: influenza activity-United States and worldwide, 2003-04 season, and composition of the 2004-05 influenza vaccine. MMWR Morb Mortal Wkly Rep 53: 547-552.

9. Nicholson KG, McNally T, Silverman M, Simons P, Zambon MC (2003) Influenza-related hospitalizations among young children in Leicestershire. Pediatr Infect Dis J 22: S228-230.
Mice were immunized intramuscularly with various doses of $\mathrm{A} /$ $\mathrm{PR} / 8 / 34, \mathrm{Ad}-\mathrm{HAl}$-con, and A/TX/05/09 HA expressing virus. Three weeks after immunization the mice were challenged intranasally with $100 \mathrm{LD}_{50}$ of influenza virus A/FM/1/47. Weight loss and death in mice immunized $10 \mathrm{vp}$ are shown in $\mathrm{A}$ and $\mathrm{B}$, respectively. Weight loss and death in mice immunized $10^{6}$ vp are shown in $\mathrm{C}$ and $\mathrm{D}$, respectively. Weight loss and death in mice immunized $10^{5} \mathrm{vp}$ are shown in $\mathrm{E}$ and $\mathrm{F}$, respectively. The mean and standard error of the control DPBS immunized mice are shown. Mice exhibiting profound signs of disease and less than $75 \%$ of baseline weights were humanely sacrificed.

(TIF)

Figure S5 Dose-dependent prophylactic responses against disease after a lethal $\mathrm{A} / \mathrm{CA} / 04 / 09$ Swine flu influenza virus challenge. Mice were immunized intramuscularly with various doses of A/PR/8/34, Ad-HAl-con, and A/ TX/05/09 HA expressing virus. Three weeks after immunization the mice were challenged intranasally with $100 \mathrm{LD}_{50}$ of influenza virus 2009 Swine Flu. Weight loss and death in mice immunized $10^{8} \mathrm{vp}$ are shown in $\mathrm{A}$ and $\mathrm{B}$, respectively. Weight loss and death in mice immunized $10^{7} \mathrm{vp}$ are shown in $\mathrm{C}$ and $\mathrm{D}$, respectively. Weight loss and death in mice immunized $10^{6}$ vp are shown in $\mathrm{E}$ and $\mathrm{F}$, respectively. Weight loss and death in mice immunized $10^{5}$ vp are shown in $\mathrm{G}$ and $\mathrm{H}$, respectively. The mean and standard error of the control DPBS immunized mice are shown. Mice exhibiting profound signs of disease and less than $75 \%$ of baseline weights were humanely sacrificed.

(TIF)

\section{Acknowledgments}

We would like to thank Mary E. Barry and Shannon May for their excellent technical assistance in the project.

\section{Author Contributions}

Conceived and designed the experiments: EAW MAB. Performed the experiments: EAW AMR RJW. Analyzed the data: EAW RJW MAB. Contributed reagents/materials/analysis tools: EAW AMR RJW. Wrote the paper: EAW MAB.

10. Potter CW, Jennings R, Clark A (1977) The antibody response and immunity to challenge infection induced by whole, inactivated and tween-ether split influenza vaccines. Dev Biol Stand 39: 323-328.

11. Clements ML, Betts RF, Tierney EL, Murphy BR (1986) Serum and nasal wash antibodies associated with resistance to experimental challenge with influenza A wild-type virus. J Clin Microbiol 24: 157-160.

12. Muszkat M, Friedman G, Schein MH, Naveh P, Greenbaum E, et al. (2000) Local SIgA response following administration of a novel intranasal inactivated influenza virus vaccine in community residing elderly. Vaccine 18: 1696-1699.

13. Muszkat M, Greenbaum E, Ben-Yehuda A, Oster M, Yeu'l E, et al. (2003) Local and systemic immune response in nursing-home elderly following intranasal or intramuscular immunization with inactivated influenza vaccine. Vaccine 21: 1180-1186.

14. Subbarao K (1999) Influenza vaccines: present and future. Adv Virus Res 54: 349-373.

15. Ennis FA, Cruz J, Jameson J, Klein M, Burt D, et al. (1999) Augmentation of human influenza A virus-specific cytotoxic T lymphocyte memory by influenza vaccine and adjuvanted carriers (ISCOMS). Virology 259: 256-261.

16. Rimmelzwaan GF, Baars M, van Amerongen G, van Beek R, Osterhaus AD (2001) A single dose of an ISCOM influenza vaccine induces long-lasting protective immunity against homologous challenge infection but fails to protect Cynomolgus macaques against distant drift variants of influenza A (H3N2) viruses. Vaccine 20: 158-163. 
17. Rimmelzwaan GF, Kuiken T, van Amerongen G, Bestebroer TM, Fouchier RA, et al. (2001) Pathogenesis of influenza A (H5Nl) virus infection in a primate model. J Virol 75: 6687-6691.

18. Rimmelzwaan GF, Osterhaust AD (2001) Influenza vaccines: new developments. Curr Opin Pharmacol 1: 491-496.

19. Webster RG, Askonas BA (1980) Cross-protection and cross-reactive cytotoxic $\mathrm{T}$ cells induced by influenza virus vaccines in mice. Eur $\mathrm{J}$ Immunol 10 : 396-401.

20. Chen MW, Cheng TJ, Huang Y, Jan JT, Ma SH, et al. (2008) A consensushemagglutinin-based DNA vaccine that protects mice against divergent H5N1 influenza viruses. Proc Natl Acad Sci U S A 105: 13538-13543.

21. Fiers W, De Filette M, El Bakkouri K, Schepens B, Roose K, et al. (2009) M2ebased universal influenza A vaccine. Vaccine 27: 6280-6283.

22. Gao W, Soloff AC, Lu X, Montecalvo A, Nguyen DC, et al. (2006) Protection of mice and poultry from lethal $\mathrm{H} 5 \mathrm{~N} 1$ avian influenza virus through adenovirusbased immunization. J Virol 80: 1959-1964.

23. Hoelscher MA, Garg S, Bangari DS, Belser JA, Lu X, et al. (2006) Development of adenoviral-vector-based pandemic influenza vaccine against antigenically distinct human H5N1 strains in mice. Lancet 367: 475-481.

24. Laddy DJ, Yan J, Corbitt N, Kobasa D, Kobinger GP, et al. (2007) Immunogenicity of novel consensus-based DNA vaccines against avian influenza. Vaccine 25: 2984-2989.

25. Ellenberger DL, Li B, Lupo LD, Owen SM, Nkengasong J, et al. (2002) Generation of a consensus sequence from prevalent and incident HIV-1 infections in West Africa to guide AIDS vaccine development. Virology 302: 155-163.

26. Gao F, Korber BT, Weaver E, Liao HX, Hahn BH, et al. (2004) Centralized immunogens as a vaccine strategy to overcome HIV-1 diversity. Expert Rev Vaccines 3: S161-168.

27. Gaschen B, Taylor J, Yusim K, Foley B, Gao F, et al. (2002) Diversity considerations in HIV-1 vaccine selection. Science 296: 2354-2360.

28. Korber B, Muldoon M, Theiler J, Gao F, Gupta R, et al. (2000) Timing the ancestor of the HIV-1 pandemic strains. Science 288: 1789-1796.
29. Nickle DC, Jensen MA, Gottlieb GS, Shriner D, Learn GH, et al. (2003) Consensus and ancestral state HIV vaccines. Science 299: 1515-1518; author reply $1515-1518$

30. Novitsky V, Smith UR, Gilbert P, McLane MF, Chigwedere P, et al. (2002) Human immunodeficiency virus type 1 subtype $\mathrm{C}$ molecular phylogeny: consensus sequence for an AIDS vaccine design? J Virol 76: 5435-5451.

31. Laddy DJ, Yan J, Kutzler M, Kobasa D, Kobinger GP, et al. (2008) Heterosubtypic protection against pathogenic human and avian influenza viruses via in vivo electroporation of synthetic consensus DNA antigens. PLoS One 3: e2517.

32. Muthumani K, Lankaraman KM, Laddy DJ, Sundaram SG, Chung CW, et al. (2008) Immunogenicity of novel consensus-based DNA vaccines against Chikungunya virus. Vaccine 26: 5128-5134

33. CDC (2009) H1N1 Flu Vaccine - Why the Delay?.

34. CDC (2009) CDC Estimates of 2009 H1N1 Influenza Cases, Hospitalizations and Deaths in the United States, April - November 14, 2009.

35. Ilyushina NA, Khalenkov AM, Seiler JP, Forrest HL, Bovin NV, et al. (2010) Adaptation of pandemic H1N1 influenza viruses in mice. J Virol 84: 8607-8616.

36. Mok H, Palmer DJ, Ng P, Barry MA (2005) Evaluation of polyethylene glycol modification of first-generation and helper-dependent adenoviral vectors to reduce innate immune responses. Mol Ther 11: 66-79.

37. Weaver EA, Lu Z, Camacho ZT, Moukdar F, Liao HX, et al. (2006) Crosssubtype $\mathrm{T}$-cell immune responses induced by a human immunodeficiency virus type 1 group $m$ consensus env immunogen. J Virol 80: 6745-6756.

38. Casimiro DR, Tang A, Chen L, Fu TM, Evans RK, et al. (2003) Vaccineinduced immunity in baboons by using DNA and replication-incompetent adenovirus type 5 vectors expressing a human immunodeficiency virus type 1 gag gene. J Virol 77: 7663-7668.

39. Weaver EA, Nehete PN, Buchl SS, Senac JS, Palmer D, et al. (2009) Comparison of replication-competent, first generation, and helper-dependent adenoviral vaccines. PLoS One 4: e5059.

40. Hirota Y, Kaji M, Ide S, Kajiwara J, Kataoka K, et al. (1997) Antibody efficacy as a keen index to evaluate influenza vaccine effectiveness. Vaccine 15: 962-967. 